\title{
Review of: "A randomised double-blind placebo- controlled clinical trial of oral hydroxyurea for transfusion-dependent $\beta$-thalassaemia"
}

sabariah md noor ${ }^{1}$

1 Universiti Putra Malaysia

Potential competing interests: The author(s) declared that no potential competing interests exist.

This manuscript is addressing an interesting and important research question. Overall, this is a clear, concise, and well-written manuscript. The introduction is relevant and theory based. Sufficient information about the previous study findings is presented for readers to follow the present study rationale and procedures. The methods are generally appropriate, and all the relevant issues were appropriately addressed. The authors make a systematic contribution to the research literature in this area of disease focus and its treatment related choices. 